\title{
Spread awareness; stop resistance
}

Wendy Thompson is an elected member of the FDI World Dental Federation Science Committee and Chair of its Antimicrobial Resistance Group. She is a member of the BDA Health \& Science Committee, and a clinical academic GDP at the University of Manchester and \{my\}dentist Kirkby Lonsdale.

The BDJ Upfront section includes editorials, letters, news, book reviews and interviews. Please direct your correspondence to the News Editor,

Kate Quinlan at k.quinlan@nature.com. Press releases or articles may be edited, and should include a colour photograph if possible.

A ccess to dental care in the UK is at crisis point. The COVID-19 pandemic has been unforgiving. From April to June 2021, four out of five people said they had difficulties accessing timely care for their dental problems. ${ }^{1}$ Healthwatch England reports that dentistry is the top issue with which it is currently dealing, with feedback from the public being nearly eight times higher than the same period in $2020 .^{1}$

This restricted access to face-to-face dental appointments resulted in a significant increase in antibiotic prescribing during 2020, which is a problem because it drives the development and spread of infections that are resistant to antibiotics. Using antibiotics to make up for a lack of access to urgent dental care is, therefore, a risk to patient safety and should be avoided wherever possible. Ensuring timely access to appropriate and effective treatment is the best way to provide care for patients with acute dental pain or infection. Healthwatch feedback tells us, however, that this is not the case currently and has not been for some time. Something fundamentally needs to change to address this.

A mismatch also exists between public expectations about NHS dentistry and the reality of dental services being provided. This is a recipe for complaints - so why is the NHS not honest with patients about what is deliverable? If the NHS does not have the resources to provide everything on the NHS website (such as Band 2 apicectomy) to all patients, then the public needs clarity about what can be expected. Is it realistic to expect clarity from government?

Managing dental pain is another area where we know public misconception exists: that antibiotics are necessary and appropriate for treating dental pain. ${ }^{2}$ While studies show that dental practitioners know that antibiotics are only required for spreading infections, they also indicate that members of the wider team are not so aware of this.
The whole healthcare system needs to facilitate guideline-congruent urgent dental care - from the most senior managers to the most junior front-line dental team members. Within the NHS, providing a conducive environment means contracting to incentivise procedures not prescriptions, and managing service provision to ensure inappropriate antibiotic use is minimised (as per the Health and Social Care Act's code of practice on the prevention and control of infections).

Keeping people with dental pain and infection safe must sit at the heart of any oral healthcare system. As the cornerstone of modern medicine (such as cancer treatments and organ transplants) and the last line of defence for some life-threatening infections, and wellbeing, poverty reduction, food security, environment and economic growth. ${ }^{3}$

For these reasons, the BDA has signed FDI World Dental Federation's pledge to reiterate its commitment to tackling AMR by preventing dental infections, optimising antibiotic stewardship and raising awareness.

Preventing infections through routine patient care is always better than curing them, as it avoids antibiotics altogether. Managing patients during COVID-19 was a significant challenge for dentistry - let's now strive to optimise antibiotic prescribing using the latest guidelines. Do you know when phenoxymethylpenicillin is indicated over amoxicillin? Spreading awareness that antibiotics don't cure toothache is

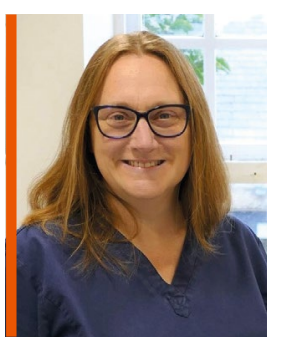

\section{'Antibiotic-resistant infections are not only a problem for those taking the antibiotics - their spread is a problem for society as a whole.'}

it is essential for the safety of our patients, friends and ourselves that we use antibiotics judiciously. In this respect, dental teams have an important role for patients' overall health and wellbeing beyond just their oral health.

Antibiotic-resistant infections are not only a problem for those taking the antibiotics - their spread is a problem for society as a whole. Within the next 30 years, more people will die from resistant infections than from cancer, unless we start acting now. The WHO predicts that antimicrobial resistance (AMR) will be the world's biggest killer by $2050 .^{3}$

The United Nations describes AMR as a silent pandemic that poses a significant threat to global sustainable development. Rising levels of AMR will hinder progress towards many of its 17 Sustainable Development Goals, particularly those focusing on health an important part of stopping resistance, including by increasing team knowledge and reducing patient expectations for antibiotics.

So what next? We know the impact that preventing oral disease can have. Perhaps the next major conversation should be a frank discussion with the public about what to expect from dentistry into the future.

World Antimicrobial Awareness Week takes place 18-24 November 2021.

\section{References}

1. BDA. BDA and Healthwatch urge Chancellor to reverse decade of cuts. Press release. 21 October 2021.

2. Emmott R, Barber SK, Thompson W. Antibiotics and toothache: a social media review. Int J Pharm Pract 2021 29: 210-217.

3. WHO. Antimicrobial resistance and the United Nations sustainable development cooperation framework: guidance for United Nations country teams. 2021.

https://doi.org/10.1038/s41415-021-3618-3 